\title{
Tag Design for RFID AC Current Sensing System
}

\author{
Irfan Ullah*, Robert Horne*, Benito Sanz-Izquierdo*, John C Batchelor* \\ *School of Engineering and Digital Arts, The University of Kent, Canterbury, UK, iu22@kent.ac.uk
}

\begin{abstract}
This study describes the development of an RFID tag system and antenna for real-time ac current sensing of individual appliances in smart homes. The operating principle of the tag system is based on the tag antenna tuning via a tuning circuit. The auto-tuning chip is embedded to compensate the antenna matching and stores the impedance tuning in the form of a 5-bit sensor code. The tag wirelessly streams the 5-bit sensor code that represents the ac current drawn by the electrical load, to the dedicated RFID reader in the range of $3 \mathrm{~m}$ at $868 \mathrm{MHz}$. The tag device is an energy harvester and a cost-effective ac current sensing solution compared to commercial smart meters in smart power metering systems. The antenna is designed to fit around the housing of the current sensor.
\end{abstract}

Index Terms-RFID sensing, self-tuning tags, Internet of Things, smart home application.

\section{INTRODUCTION}

There is an increase in demand for the enrolling of smart electricity meters in homes for measuring real-time electricity consumption of electrical appliances in smart power metering systems [1]. Smart meters record the electricity usage and wirelessly stream data to an in-home display, which can aid the household to track the power consumption of electrical appliances. Tracking the power consumption of multiple individual appliances in homes can help households to prevent unnecessary energy usage. Enhancing energy-saving smart technologies will have an impact to reduce carbon emissions and cut energy costs for households [2].

There has been an RFID tag device for real-time ac current sensing of individual appliances in smart homes in which a self-tuning tag linked with a tuning circuit is integrated with a Current Transformer (CT) [3]. However, the tag antenna design is not well integrated with the CT housing, which makes the overall size of the tag device comparatively large. As a result the design is not suitable for deployment as a compact device. Furthermore, there are some commercial ac current sensors available serving wireless sensing devices. They have integrated CT into an energy monitoring unit with the onboard high gain antenna. Such as, a NCD long-range IoT wireless ac current monitor [4] is designed to measure ac current in the range of $0-100 \mathrm{~A}$. The sensor is integrated with a monopole antenna operating at $868 \mathrm{MHz}, 900 \mathrm{MHz}$ and $2.4 \mathrm{GHz}$. However, the sensor is suitable for industrial uses and is cost-intensive to use in domestic applications and needs an external power source. Similarly, an Alta wireless ac current meter is designed to measure ac current up to 20 A [5], embedded with a monopole antenna working at $868 \mathrm{MHz}$ and $900 \mathrm{MHz}$. The meter uses a 3-3.8 V battery which increases the maintenance cost. Alternatively, Pressac wireless current sensor [6] is a passive sensor for measuring real-time energy usage, which communicates data via the EnOcean wireless radio protocol. The sensor required a smart gateway to wirelessly transmit data via WiFi or LTE (4G) to the consumer access device.

Therefore, this paper presents an RFID sensing tag and antenna for ac current sensing in a power metering system at smart homes. The tag antenna is well integrated with the CT housing. The antenna is designed to be linked with the varactor circuit and connected with the CT terminals. Measurements show that the tag device can sense current in the range of 1-13 A with a resolution of $0.5 \mathrm{~A}$ and read range of 3-3.5 m across all current reading at $868 \mathrm{MHz}$. Furthermore, the overall size of the tag device is reduced to half as compared to the size of tag system proposed in [3]. The tag device can be used as an ac current sensor at the domestic level because of its simple structure when compared to the expensive alternative smart meters.

\section{TAG SYSTEM}

The tag system consists of: (i) a current transformer, (ii) a varactor circuit and (iii) a self-tuning RFID tag and antenna, as shown in Fig. 1. The CT is used to measure ac current flow in an electrical wire and transform it proportionally into a dc voltage. The CT supplies the dc to the varactor tuning circuit. The tuning circuit capacitance varies as a function of reverse bias voltage from the CT and is connected to the tag antenna input. The combined antenna/CT impedance is compensated by the auto-tuning chip and the results are translated to a 5-bit sensor value. This sensor value is related to the load current as the primary current flow has a linear relationship to the secondary windings of the CT. The tag wirelessly streams the sensor value to the external RFID reader at $868 \mathrm{MHz}$.

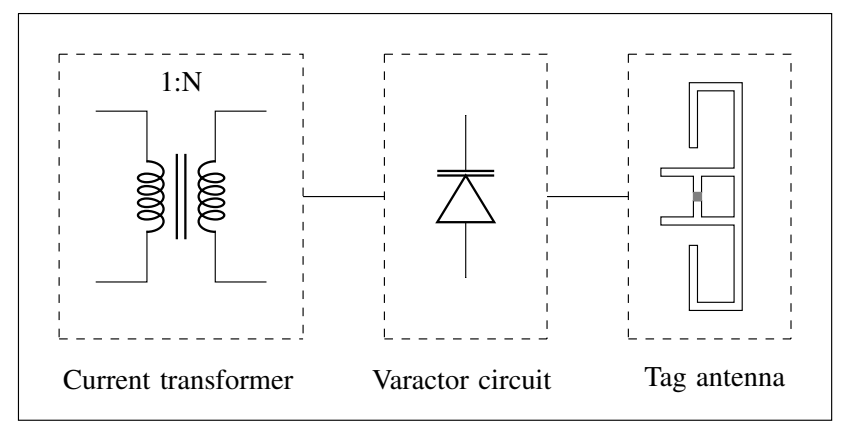

Tag system

Fig. 1. Block diagram of the RFID tag system for ac current sensing. 


\section{A. Current Transformer (CT)}

A split-core current transformer [7] is used to measure the primary input current range from 1-13 A and convert into $1-13 \mathrm{~V} \mathrm{dc}$ proportionally with the full-scale accuracy of $\pm 2 \%$ in $50 / 60 \mathrm{~Hz}$ operating speed. The split-core CT is installed around the existing power cable without interrupting the existing circuit. The output terminals (M3 screw) of the $\mathrm{CT}$ are connected to the output leads (varactor circuit).

\section{B. Varactor circuit}

The varactor circuit is composed of two inductors, a varactor diode and two capacitors, as depicted in Fig. 2. The inductors are used as RF choke and to isolate the copper tracks from the tag antenna. The tuning diode [8] is reverse biased and controlled by the dc voltage from the $\mathrm{CT}$. The total capacitance of the tuning diode lies between $0.30-2.22 \mathrm{pF}$ when the bias voltage changes from $0-20 \mathrm{~V}$. The dc voltage is isolated from the chip via $15 \mathrm{pF}$ capacitors. The dimensions of the tuning circuit are shown in Fig. 3(b). Two $11.75 \mathrm{~mm}$ long and $1 \mathrm{~mm}$ wide copper tracks with a $6 \mathrm{~mm}$ gap connect the dc voltage from the CT. The circuit is designed for 0603 size components.

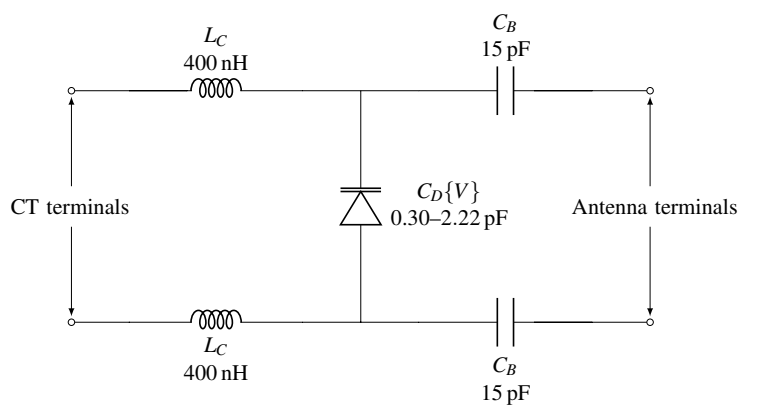

Fig. 2. Schematic diagram of the varactor tuning circuit linked with the tag antenna terminals.

\section{RFID tag antenna design}

The tag antenna was designed in a square form factor with a hallow central space to achieve a compact form integration with the CT, as shown in Fig. 3(a). The antenna was simulated in Computer Simulation Technology (CST) Microwave Studio [9] on copper Mylar sheet with a dielectric constant $\left(\varepsilon_{r}\right)$ of 2.8 , loss tangent $(\delta)$ of 0.003 and thickness of $0.18 \mathrm{~mm}$ including the copper thickness of $0.04 \mathrm{~mm}$. An Axzon Magnus S2 chip [10] with the characteristic impedance of $2-3 \mathrm{pF}$ in parallel with $2284 \Omega$ was embedded to the tag antenna ports. The total capacitance of the chip for the tag antenna is:

$$
C_{T}(n)=C_{\min }+n C_{o}
$$

where $C_{T}(n)$ is the total capacitance, $C_{\min }$ is the minimum capacitance of $2 \mathrm{pF}$ of the chip, $\mathrm{n}$ is the sensor code from $0-31$ and $C_{o}$ is a tunable step within the $1 \mathrm{pF}$ tunable range. Therefore, for simulation the chip impedance of was fixed to $1.63-\mathrm{j} 61.07 \Omega$, at $868 \mathrm{MHz}$. The tag was mounted at the $6 \mathrm{~mm}$ above the bottom of the CT, as shown in Fig. 4 and was simulated into two configurations, (i) the tag with a $0.3 \mathrm{pF}$ capacitor soldered across the antenna terminals, (ii) the tuning circuit, Fig. 3(b), was attached at the tag antenna terminals (excluding the $0.3 \mathrm{pF}$ capacitor). The total capacitance of the varactor circuit was set to $0.3 \mathrm{pF}$, in accordance with:

$$
\frac{1}{C_{e q}}=\frac{1}{C_{B}}+\frac{1}{C_{D}\{V\}}+\frac{1}{C_{B}}
$$

where $C_{e q}$ is the total equivalent capacitance of the varactor circuit, $C_{B}$ is the dc block capacitor of $15 \mathrm{pF}$ and $V_{D}\{V\}$ is the capacitance of the tuning diode as a function of a reverse bias voltage [8].

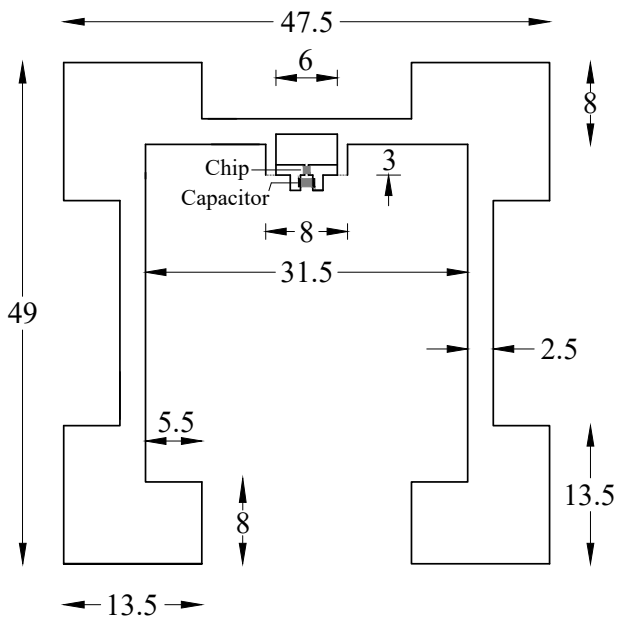

(a)

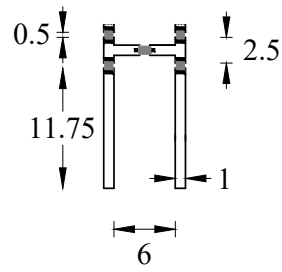

(b)

Fig. 3. (a) The tag antenna design geometry, (b) varactor tuning circuit. (All dimensions in $\mathrm{mm}$ )

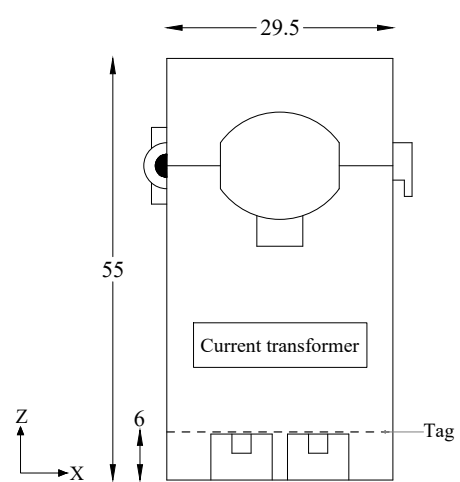

(a)

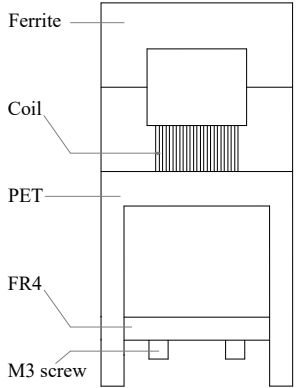

(b)
Fig. 4. A current transformer design used for simulations in CST (a) CT outside housing, (b) CT inside components. (All dimensions in $\mathrm{mm}$ ) 
The simulated antenna reflection coefficient with a $0.3 \mathrm{pF}$ capacitor and the tag coupled with the tuning circuit are illustrated in Fig. 5. The antenna in both configurations has a good match at UHF RFID band (865-868 MHz). Fig. 6 displays the radiation patterns of the tag configurations with a realized gain at $868 \mathrm{MHz}$ of $0.159 \mathrm{~dB}$ and $-0.455 \mathrm{~dB}$, respectively. Additionally, the tag simulated radiation efficiency was $-0.9 \mathrm{~dB}$ and the radiation efficiency of the tag with the varactor tuning circuit was $-0.7 \mathrm{~dB}$.

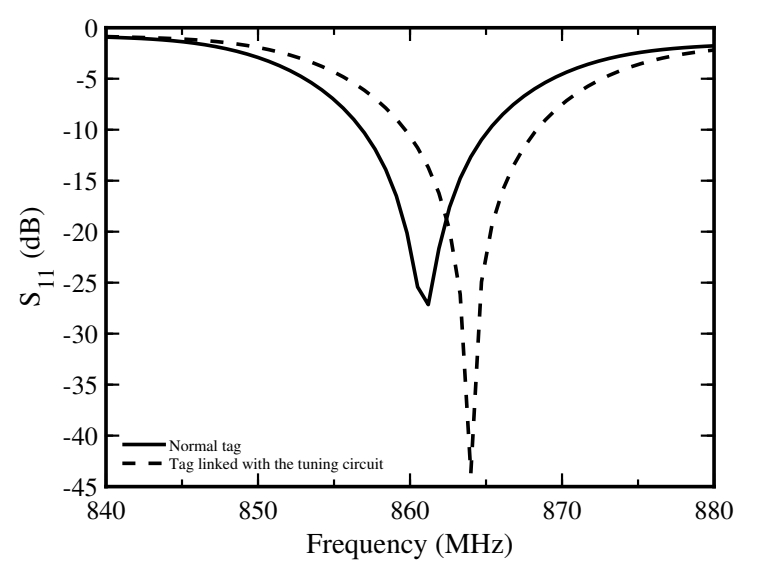

Fig. 5. Simulated reflection coefficient of the RFID tag design.

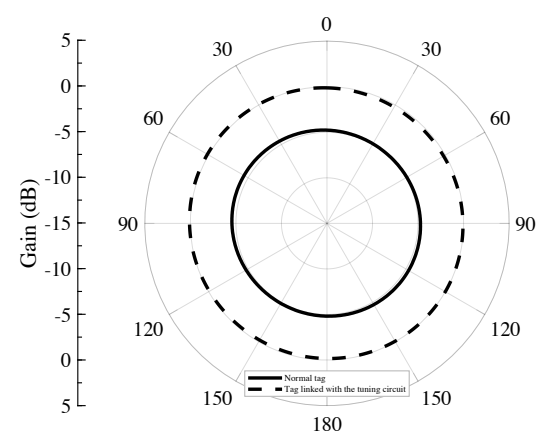

(a)

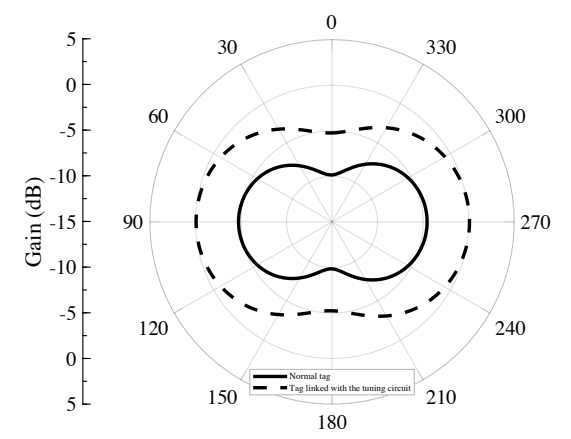

(b)

Fig. 6. Polar plot of CST simulated tag system realized gain (a) $y-z$ plane (b) azimuth plane.

\section{MEASUREMENTS AND RESULTS}

\section{A. Tag: sensor code and read range}

In the first experiment, the antenna was etched onto Mylar film, of copper thickness $0.18 \mathrm{~mm}$, Fig. 3(a). A $0.3 \mathrm{pF}$ capacitor was soldered across the antenna terminals and mounted on the CT. The UHF reader Tagformance Pro system [11] was used to measure performance. The tag was placed $30 \mathrm{~cm}$ away from a linear polarized antenna of $6 \mathrm{dBi}$ gain. Fig. 7 illustrated the measured sensor code of the tag. The sensor code of the tag is fine-tuned to $868 \mathrm{MHz}$ to achieve the maximum sensing range $(0-31)$ of the tag. The read range of the tag across different frequencies (Fig. 7) and a good read range of about $3 \mathrm{~m}$ is observed at the UHF band $(860-868 \mathrm{MHz})$.

\section{B. AC current sensing setup}

In the final experiment, the tag was linked with the tuning circuit and fabricated on Mylar film. The lumped elements were soldered across the slots. The end of the copper tracks of the tuning circuit was connected to the output terminals of the CT via M3 screw, Fig. 8. The tag was tested with the Tagformance Pro system as described earlier and the reader

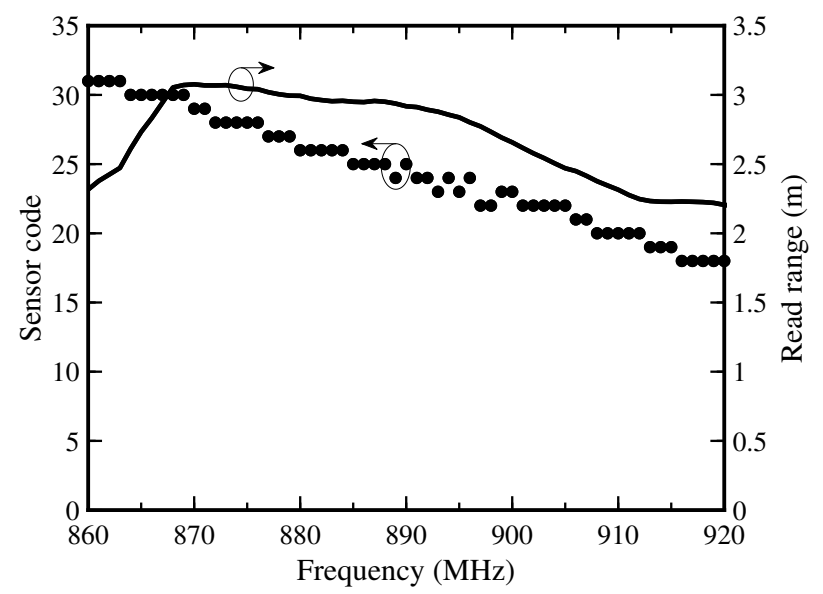

Fig. 7. Measured sensor code and read range of the tag with a $0.3 \mathrm{pF}$ capacitor across the antenna terminals.

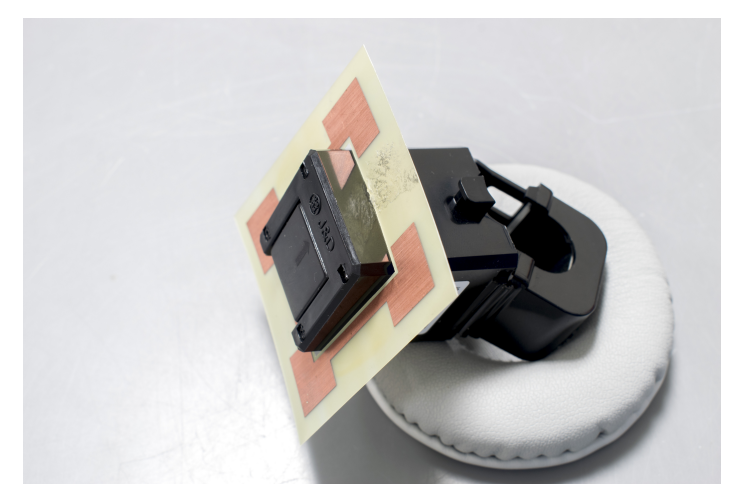

Fig. 8. A prototype of the RFID tag system. 


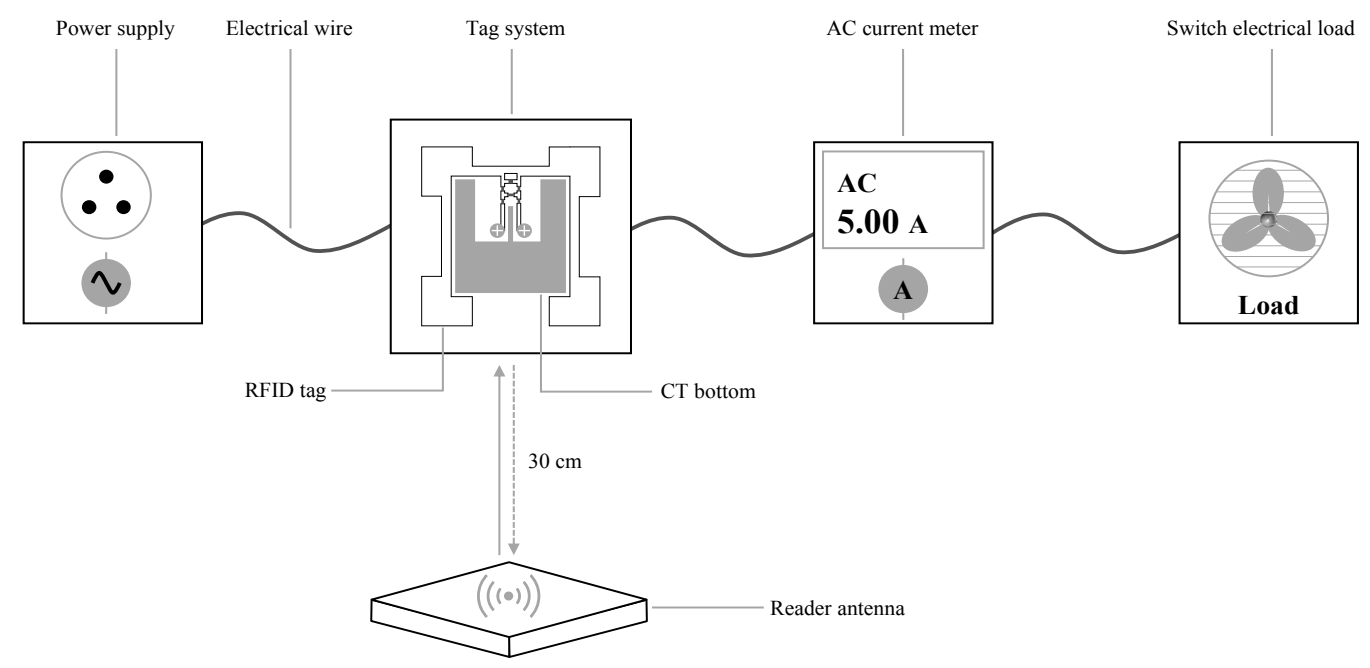

Fig. 9. Set up for ac current sensing.

antenna was placed $30 \mathrm{~cm}$ away from the tag, as shown in Fig. 9. The CT was clamped around a single electrical conductor and the split-core part of the CT was enclosed in an insolated box. A switchable electrical load was used to draw ac current from 0-13 A and the electrical load was powered from a $240 \mathrm{~V}$ ac power outlet with the operating frequency of 50/60 Hz. A current meter [12] was included in the set up to measure the ac current drawn by the electrical load and standardized the sensor code of the tag device.

\section{Current and sensor code relationship}

The ac current measurements were performed from 0-13 A with a step of $0.5 \mathrm{~A}$ and each measurement was made 10 times for a single tag. Fig. 10 illustrates the measured sensor code and is compared with the ac current flow in the power cable to an electrical load. The measurements result shows that the tag system can detect ac current in the range of 1-13 A with a resolution of $0.5 \mathrm{~A}$. At $0 \mathrm{~A}$ the output capacitance of the tuning circuit is maximum and therefore the tag is detuned from $868 \mathrm{MHz}$. The tag device response is saturated at $12 \mathrm{~A}$, because the reactance of the tuning diode becomes saturated when the bias voltage reached to $12 \mathrm{~V}$. However, the input current sensing range of the tag devices can be increased above $13 \mathrm{~A}$ by adjusting the tuning circuit of the system. Furthermore, Fig. 11 shows the measured read range of about 3-3.5 m of the tag across all current reading at $868 \mathrm{MHz}$. The lowest read range of $1.4 \mathrm{~m}$ was recorded at 0 A because the tuning circuit reactance is at its peak and detune the tag from $868 \mathrm{MHz}$. The tag was placed at a different distance away from the reader antenna to assess if non-linearities occurred at short range. At $2 \mathrm{~cm}$ the tag response was 22, which is just 2 sensor values less than the value measured at $30 \mathrm{~cm}$. Additionally, the distance between the tag and the reader antenna was gradually increased and at $7 \mathrm{~cm}$ the tag response returned to the original value for $30 \mathrm{~cm}$. The effect of distance on the sensor value of the tag system can be seen in Fig. 12.

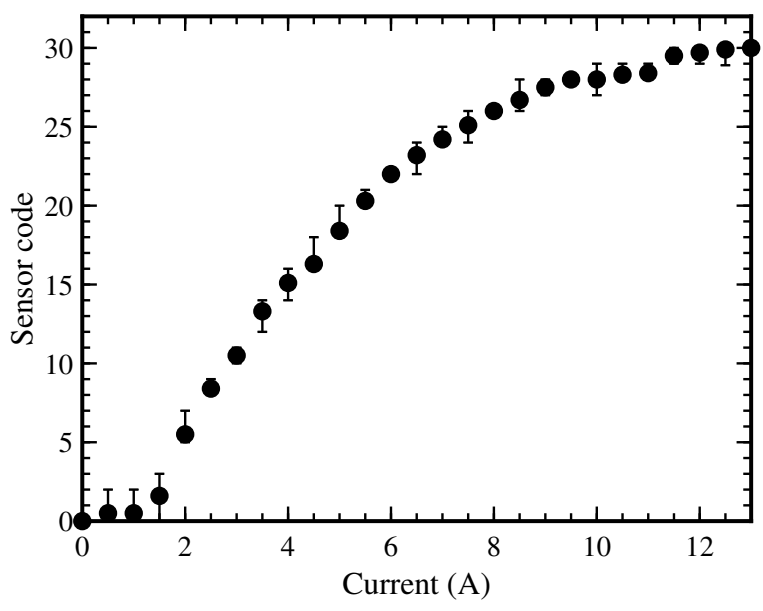

Fig. 10. Measured sensor code of the tag system at $868 \mathrm{MHz}$.

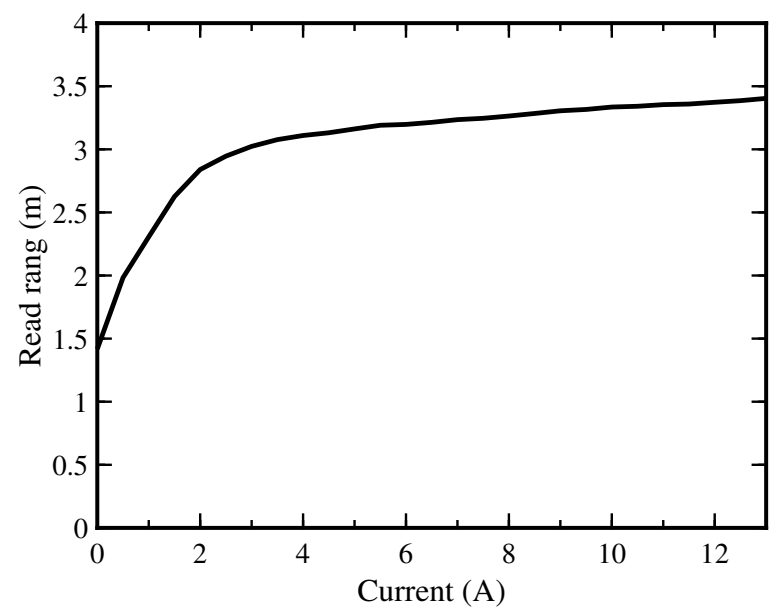

Fig. 11. Measured read range of the tag system at $868 \mathrm{MHz}$. 


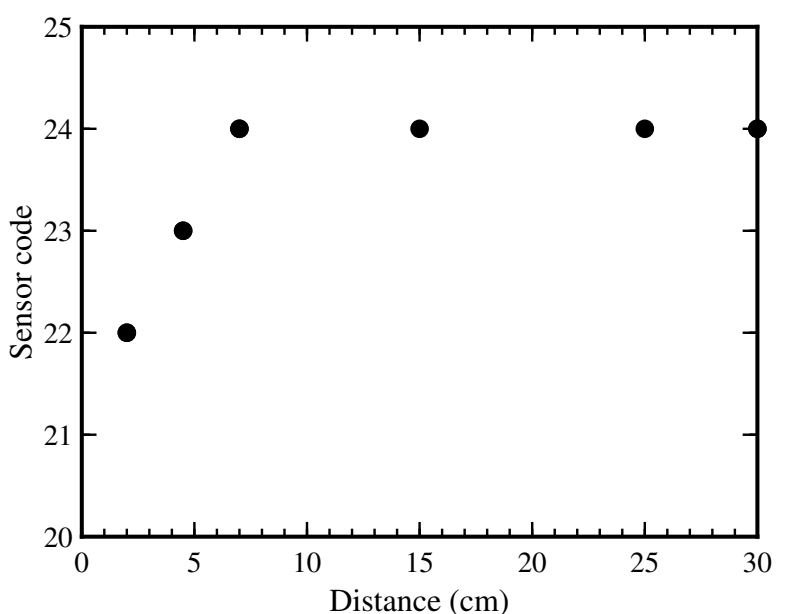

Fig. 12. The effect of distance variation between the tag system and the reader antenna on the sensor code of the tag system at $868 \mathrm{MHz}$.

\section{CONCLUSION}

In this paper, we demonstrated a new UHF RFID tag design and antenna for ac current transformer for measuring realtime ac current in smart power metering systems. The tag and antenna were experimentally tested with a switchable electrical load and the measured results show that the tag can detects ac current in the range of 1-13 A with a resolution of $0.5 \mathrm{~A}$ and has a good read range of between 3 and $3.5 \mathrm{~m}$ through all current readings at $868 \mathrm{MHz}$. However, the input current sensing range of the tag devices can be increased above $13 \mathrm{~A}$ by adjusting the tuning circuit of the system. The tag system has a very simple structure compared to microcontrollerbased smart electricity metering and can be used for real-time monitoring of power consumption of multiple appliances with a single RFID reader at smart homes.

\section{ACKNOWLEDGMENT}

The INCASE project is part-funded by the European Commission as part of the 2 Seas Intereg V Programme. We would like to thank Antonio Mendoza for fabricating the tags.

\section{REFERENCES}

[1] Government "SK, "Smart meters: unlocking https://www.gov.uk/government/publications/smart-meters-unlockingthe-future/smart-meters-unlocking-the-future

[2] International Energy Agency (2019, Sept. 13). World energy outlook 2018: The gold standard of energy analysis [Online]. Available: https://www.iea.org/weo2018/

[3] I. Ullah, R. Horne, B. Sanz-Izquierdo and J. C. Batchelor, "RFID AC Current Sensing Technique," in IEEE Sensors Journal. doi: 10.1109/JSEN.2019.2949856

[4] NCD (2019, Aug. 26). IoT long range wireless ac current monitor [Online]. Available: https://store.ncd.io/product/wireless-ac-current-monitor/

[5] Monnit (2019, Aug. 26). Alta wireless ac current meter - 20 A - AA battery powered [Online]. Available: https://www.monnit.com/Product/MNS2-9-W2-CM-020/

[6] Pressac (2019, Aug. 26). Current sensor: one channel [Online]. Available: https://www.pressac.com/current-monitoring-sensors/
[7] Magnelab (2019, Sept. 10). DCT-0016-10/10Vdc split-core current sensor [Online]. Available: https://www.magnelab.com/products/ac-splitcorecurrent-sensor-dct-0016-100/

[8] Skyworks, "SMV2019 to SMV2023 series: hyperabrupt junction tuning varactors," SMV2019 to SMV2023 varactors datasheet, Aug. 2015.

[9] CST Microwave Suite 2017. [Online]. Available: https://www.cst.com

[10] Axzon (2019, Jul. 5). [Online]. Available: https://axzon.com

[11] Voyantic (2019, Sept. 10). Tagformance Pro [Online]. Available: https://voyantic.com/products/tagformance-pro

[12] Brennenstuhl smart technology (2019, Sept. 9). Primera-line wattage and current meter PM $231 \mathrm{E}$ [Online]. Available: https://www.brennenstuhl.co.uk/en-GB/products/adapter-plugs/primeraline-wattage-and-current-meter-pm-231-e-gb 